\title{
Image Segmentation Techniques in Bone Structure Psychiatry
}

\author{
${ }^{1}$ Ajayi Olayinka Adedoyin*, ${ }^{2}$ Olamide Timothy Tawose, ${ }^{3}$ Olu Sunday Adetolaju \\ $1,2,3$ \\ Ekiti State University, Ado-Ekiti, Nigeria
}

\begin{abstract}
:
Today, a large number of x-ray images are interpreted in hospitals and computer-aided system that can perform some intelligent task and analysis is needed in order to raise the accuracy and bring down the miss rate in hospitals, particularly when it comes to diagnosis of hairline fractures and fissures in bone joints. This research considered some segmentation techniques that have been used in the processing and analysis of medical images and a system design was proposed to efficiently compare these techniques. The designed system was tested successfully on a hand X-ray image which led to the proposal of simple techniques to eliminate intrinsic properties of x-ray imaging systems such as noise. The performance and accuracy of image segmentation techniques in bone structures were compared and these eliminated time wasting on the choice of image segmentation algorithms. Although there are several practical applications of image segmentation such as content-based image retrieval, machine vision, medical imaging, object detection, recognition tasks, etc., this study focuses on the performance comparison of several image segmentation techniques for medical X-ray images.
\end{abstract}

Keywords: Medical Images, Segmentation Techniques, Hairline Fracture, Bone Structures.

\section{Introduction}

In medical imaging, diagnosis and treatment of bone fractures and fissures, it is important to get the initial findings right. Such precision, or the lack thereof, could mean the difference between a successful treatment of such medical issues or prolonged pain for the patient. As opposed to images used in other areas of life, when examining medical images, it is very Important to identify and separate objects in areas of interest from the background [1] and the rest of the image [1] [2]. This task can be realized through segmentation. According to [2], image segmentation remains one of the most challenging issues, and one of the most active areas of image processing domain. Segmentation attempts to divide an image into regions such that attributes remain constant for each region but those attributes differ from attributes of other regions. It is based on that reasoning that Zhou et al. defined image segmentation as an exhaustive partitioning of an input image into regions, each of which is considered to be homogeneous with respect to some image property of interest e.g., intensity, color, or texture [1]. For example, if an image has strong edges, edge detection techniques can be used to partition the image into its components using those edges. As an illustration of segmentation given in [1], Figure 1 shows how some anatomical structures are separated from the image of a stained mouse embryo. Figure 1a shows a stained image of a stained mouse embryo at day 14 and Figure 1b shows Segmented image of (a), where heart, liver and kidney had been identified and marked by color red, yellow and green.
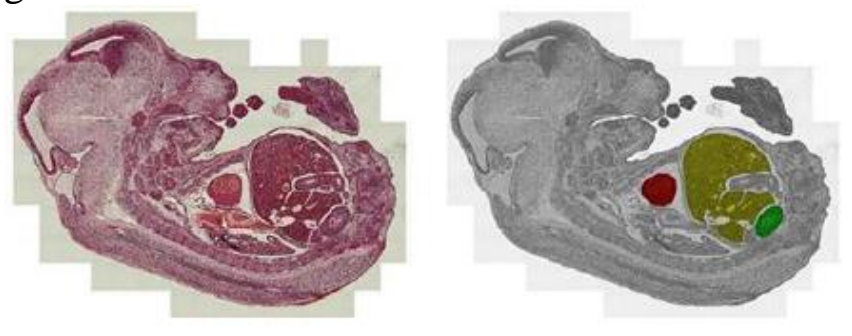

(a) The original image (b) Image segmentation

Figure 1: Basic Image segmentation [1]

In summary, the goal of segmentation is to extract useful information from images in medical imaging applications [2]. The quality of medical imagery has improved in recent times due to advances in modern science. The history of medical imaging began with the invent of 
Roentgen rays (X-rays), after which came inventions such as Magnetic Resonance Imaging (MRI), Ultrasound (US), Computed Tomography (CT), Nuclear Imaging, including Single Photo Emission Computed Tomography (SPECT), and Position Emission Tomography (PET) [2]. In recent times, segmentation techniques on medical images has been applied to anatomical structures, tumors and fractures localization, diagnosis and treatment, planning, computer integrated surgery, tissue classification, or tumor volume estimation [2].

There is much complexity to be considered when considering

Segmentation of X-ray images, as they may be affected by external factors such as noise. These factors could cause the regions of Interest (ROI) to become indistinct or disconnected. Other factors that may also affect X-rays are resolutions of the original images and the illumination of such images, which is most likely caused by the environment or the equipment used to capture the originals. As discussed in [2], it is important to consider specially bone regions in X-rays, as they can be confused with other bones or some other parts of the human skeletal system that may appear in X-rays.

\section{Important Terminologies}

\subsection{Feature Extraction}

Feature extraction is the process of reducing the segmented image into few numbers or sets of numbers that dene the relevant features of the image. These features must be carefully chosen in such a way that they are a good representation of the image and encapsulate the necessary information. Some examples of features can be image properties like the mean, standard deviation, gradient and edges. Generally, a combination of features is used to generate a model for the images. Cross validation is done on the images to see which features represent the image well and those features are used. Features can sometimes be assigned weights to signify the importance of certain features. For example, the mean in a certain image may be given a weight of 0.9 because it is more important than the standard deviation which may have a weight of 0.3 assigned to it. Weights generally range from 0 to 1 and they define how important the features are. These features and their respective weights are then used on a test image to get the relevant information.
To classify the bone as fractured or not, Peng [3] measures the neck-shaft angle from the segmented femur contour as a feature. Texture features of the image such as Gabor orientation (GO), Markov Random Field (MRF) and intensity gradient direction (IGD) are used by Lum et al [4] to generate a combination of classifiers to detect fractures in bones. These techniques are also used by Lim et al to look at femur fractures specifically. Best parameter values for the features can be found using various techniques.

\subsection{Classifiers and Pattern Recognition}

After the feature extraction stage, the features have to be analyzed and a pattern needs to be recognized. For example, the features mentioned above like the neck-shaft angle in a femur X-ray image need to be plotted. The patterns can be recognized if the neck-shaft angles of good femurs are different from those of fractured femurs. Classifiers like Bayesian classifiers and Support Vector Machines are used to classify features and find the best values for them. For example, Lum et al [5] used a support vector machine called the Gini-SVM and found the feature values for GO, MRF and IGD that gave the best performance overall. Clustering, nearest neighbour approaches can also be used for pattern recognition and classification of images. For example, the gradient vector of a healthy long bone $\mathrm{X}$-ray may point in a certain direction that is very different to the gradient vector of a fractured long bone X- ray. So, by observing this fact, a bone in an unknown $\mathrm{X}$-ray image can be classified as healthy or fractured using the gradient vector of the image [2] [5].

\subsection{Thresholding and Error Classification}

Thresholding and Error Classification is the final stage in the digital image processing system. Thresholding is one of the basic segmentation techniques [2] [6]. Thresholding an image is a simple technique and can be done at any stage in the process. It can be used at the start to reduce the noise in the image or it can be used to separate certain sections in an image that has distinct variations in pixel values. Thresholding is done by comparing the value of each pixel in an image and comparing it to a threshold. The image can be separated into regions or pixels that are greater or lesser than the threshold value. Multiple thresholds can be used to achieve thresholding with many levels [6].

Given an image I, thresholding method tries to 
find a threshold $t$ such that pixels with intensity values greater than or equal to $t$ are categorized into group 1, and the rest of the pixels into group 2.Thresholding requires that the intensity of the image has a bimodal distribution. The algorithm can perform well on simple images with bimodal intensity distribution [6]. However, most of the medical images do not have bimodal distribution of intensity [7]. In this case, thresholding result cannot correctly partition the images into various anatomical structures as shown in Figure 2.

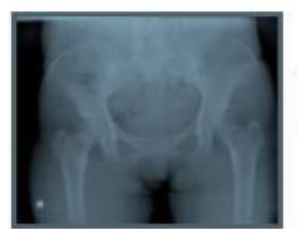

(a)

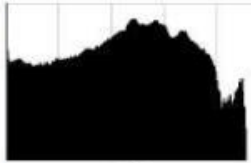

(b)

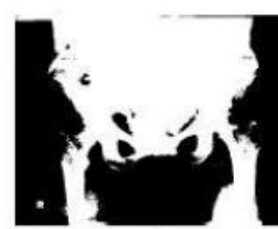

(c)
Figure 2: Thresholding of image with multimodal intensity distribution. (a) Input image. (b) Intensity histogram of (a). (c) Result of thresholding at $t=127$. [7]

Otsu's method [8] is a way of automatically thresholding any image. Otsu's method uses the variance of the image to determine the threshold. Otsu's method finds this threshold value, $t$ that minimizes the variance, $\sigma^{2}(t)$, within the two pixel set, one set with values greater than $t$ and the other with values less than $t$. The variance $\sigma^{2}(t)$, can be calculated as:

$\sigma^{2}(t)=\eta_{1}(t) \sigma_{1}^{2}(t)+\eta_{2}(t) \sigma_{2}^{2}(t)$

where $\sigma_{1}^{2}(t)$ and $\sigma_{2}^{2}(t)$ are the variances of the two pixel classes and $\eta_{1}(t)$ and $\eta_{2}(t)$ : are the number of pixels in each class [8].

Thresholding of an image can be done manually by using the

histogram of the intensities in an image [9]. It is difficult to threshold noisy images as the background intensity and the foreground intensity may not be distinctly separate. Figure 2.3, shows an example of an image and its histogram that has the pixel intensities on the horizontal axis and the number of pixels on the vertical axis [9].

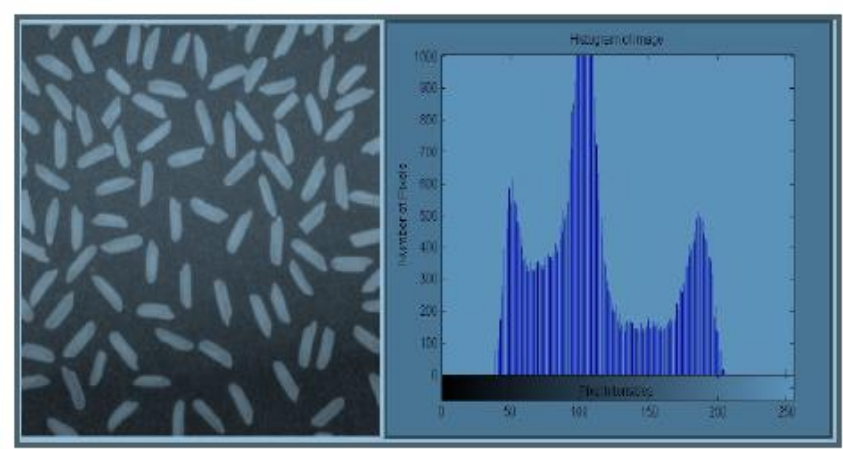

(a) The original image

(b) The histogram of the image

Figure 3: Histogram of image [9]

Figure 3 shows the original image and the histogram of the image. Figure 4, shows thresholding done on the image displayed in Figure 3. Figure $4 \mathrm{a}$ is the original image, Figure $4 \mathrm{~b}$ is the image using a threshold of 50. So the pixel values that are greater than 50 are set to 1 and those less than 50 are set to

0 . As 0 and 1 are shown as black and white respectively, and as most of the image has pixel values greater than 50 (according to the histogram in Figure 3b.), the image in Figure $4 \mathrm{~b}$ is mostly white. The image in Figure $4 \mathrm{c}$ is the original image thresholded with a value of 150 . Here the pixel values that are less than 150 are set to 1 and the ones greater than 150 are set to 0 . This is the opposite of what is done in Figure $4 \mathrm{~b}$ and is done to show that the grains of rice can be separated from the image in this way. The final image, Figure $4 \mathrm{~d}$, is a combination of two thresholds applied to the original image. So it is a combination of the images Figure $4 \mathrm{~b}$ and Figure 4c. This is multi-level thresholding as it has two threshold levels.

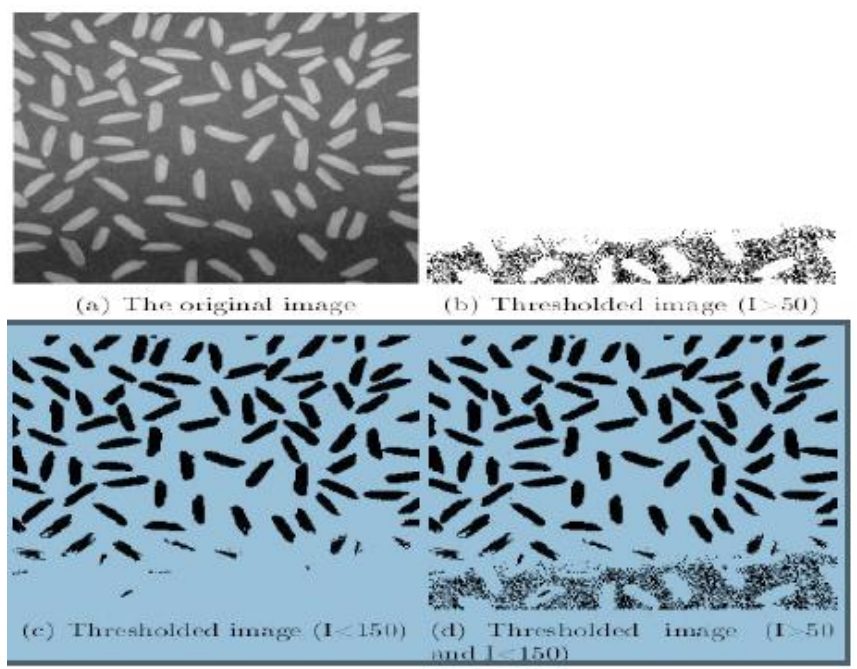

Figure 4: Thresholding in images [9]

\subsection{Edge-based Segmentation Algorithms}


Another segmentation technique is Edge-based segmentation algorithms. These algorithms use edge detectors to find edges in the image. According to [10], Traditional Sobel edge detector uses a pair of 3-by-3 convolution kernels to compute the first order derivatives (gradients) along the $\mathrm{x}$ - and $\mathrm{y}$ - directions of the 2-D image. Instead of computing first order derivatives, the Laplacian computes the second order derivatives of the image. Usually, the Laplacian is not applied directly on the image since it is sensitive to noise. It is often combined with a Gaussian smoothing kernel, which is referred to as the Laplacian of Gaussian function [11].

Other notable techniques for Image segmentation discussed in [11] are Region-based segmentation algorithms, including region growing and watershed; Graph-based approach, with modifications [12]; Good/Bad Classification technique [13]; another classification-based technique that uses Fuzzy reasoning methods proposed to detect the cardiac boundary automatically [14]; Deformable model including Active Contour Models (Snakes) [15]; level set approach [16]; and finally, the Active Shape / Appearance Model (ASM/AAM) [17].

\section{Motivations}

Conventionally, doctors in hospitals examine the bone $\mathrm{x}$-ray images based on their experience and knowledge whether a fracture exist. These kinds of manual inspection of $\mathrm{x}$-rays consume a lot of time and the process itself is monotonous and mistakes might be made during the inspection. These sort of error could mean the difference between health or prolonged pain for patients. The specific objectives of the study are to:

i. Provide simple techniques to eliminate intrinsic properties of x-ray imaging systems

ii. Compare the performance and accuracy of image segmentation techniques

iii. Analyze the effect of different algorithms on image segmentation of bone structures.

\section{Methodology}

The research is designed to proceed through the concurrent steps. In summary, the Digital X-ray images used are gotten from the Internet. Image enhancement is then performed on the image through histogram equalization techniques. Intrinsic properties of x-ray imaging systems such as noise are then eliminated through preprocessing techniques and then application of image segmentation algorithms on the image to make a comparative analysis.

The creation of a logical diagram which portrays the steps involved in the system design is derived from the architectural diagram shown below. The creation of a physical system design that lists the algorithms and methods needed to perform the steps in the logic design are summarized.

Figure 5 presents the system logic design indicating the flow of processing in the proposed scheme.

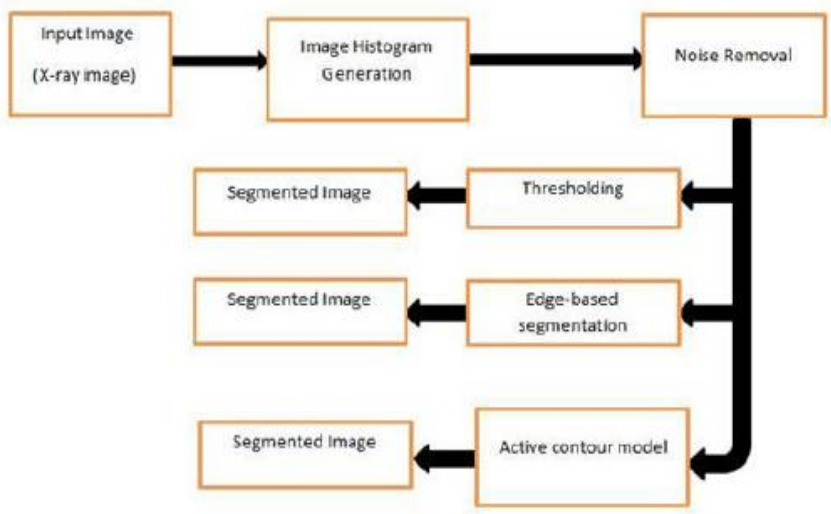

\section{Figure 5: Proposed Scheme (System block diagram)}

Figure 6 presents the robust system flow for the effective performance comparison of image segmentation techniques and the activities involved in each step are explicitly explained in the sub-sections that follow.

\section{The Input Image}

The input image is a Digital X-ray image. Image is replica of an object. An image defined in the "real world" is considered as a two dimensional function $F(x, y)$, where $x$ and y are spatial (plane) coordinates and the amplitude of $F$ at any pair of coordinates $(x, y)$ is called the intensity or gray level of the image at that point [18].

\section{Image Histogram Processing}

Histogram of an image represents the relative frequency of occurrence of various gray levels in the image. The histogram of a digital image with gray levels in the range $[0, L-1]$ is a discrete function

$$
\mathrm{h}\left(r_{k}\right)=n_{k}
$$

Where $r_{k}$ is the $k^{\text {th }}$ gray level/intensity value and $n_{k}$ is the number of pixels in the image having gray level $r_{k}$. 

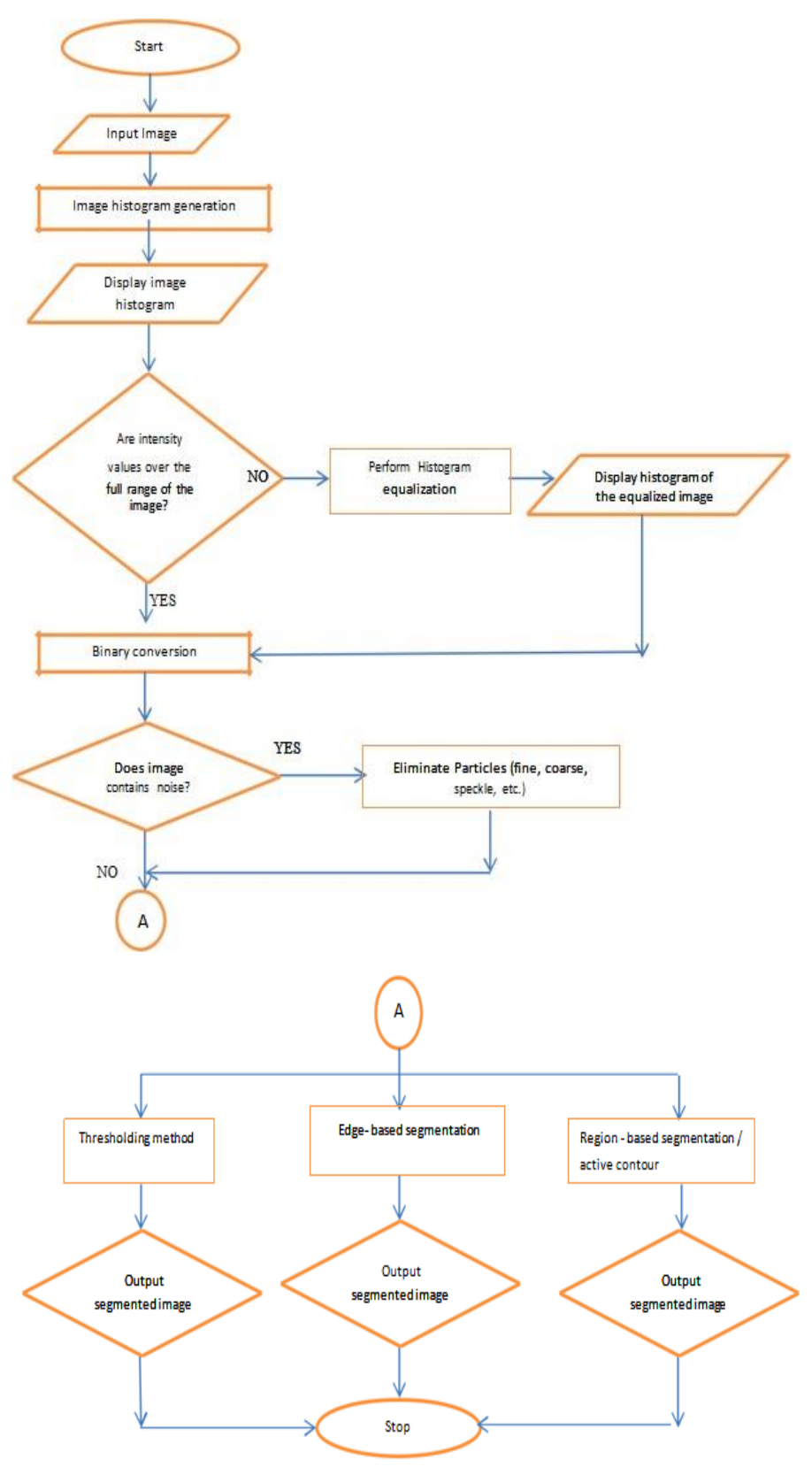

Figure 6: System flow design of the proposed scheme

Histograms are frequently normalized by the total number of pixels in the image.

Assuming an $M \times N$ image, a normalized histogram is given by:

$p\left(r_{k}\right)=n_{k} / M N \quad$ (3) for $k=0,1, \ldots, L-1$.

$p\left(r_{k}\right)$ gives an estimate of the probability of occurrence of gray level $r_{k}$. The sum of all components of a normalized histogram is equal to 1 [18]. The shape of the histogram of an image gives us useful information about the possibility for contrast enhancement.

\section{Histogram Equalization}

Histogram equalization is a common technique for enhancing the appearance of images. Histogram equalization is an entirely automatic procedure that requires no user input. The objective is to map an input image to an output image such that its histogram is uniform after the mapping. The histogram equalization process can be used to spread the intensity values over the full range of the image. The equalization process works in the steps we describe below [9] [18] [19]:

a) Let $r$ represent the gray levels in the image to be enhanced and $s$ is the enhanced output with a transformation of the form $s=\mathbf{T}(r)$.

b) Assumption:

1. $\mathbf{T}(r)$ is single-valued and monotonically increasing in the interval $[0,1]$, which preserves the order from black to white in the gray scale.

2. $0 \leq \mathrm{T}(\mathrm{r}) \leq 1$ for $0 \leq \mathrm{r} \leq 1$, which guarantees the mapping is consistent with the allowed range of pixel values.

3. If $\mathrm{P}_{\mathrm{r}}(r)$ and $\mathbf{T}(r)$ are known and $\mathbf{T}^{-1}(s)$ satisfies condition (i), the probability density function (pdf) of the transformed gray levels is

$$
\mathrm{P}_{s}(s)=\left.\mathrm{P}_{r}(r) \frac{d r}{d s}\right|_{r=T^{-1}(s)}
$$

$\mathrm{r}=\mathbf{T}^{-1}(\mathrm{~s})$ is the inverse transformation from $s$ back to $r$

4. If $s=\mathrm{T}(r): \int_{0}^{r} \mathrm{P}_{r}(w) d w:=$ mfor $0 \leq r \leq 1$, then we have

$$
\frac{d s}{d r}=\mathrm{P}_{\mathrm{r}}(r)
$$

and hence $\mathrm{P}_{s}(s)=1$ for $0 \leq s \leq 1$

5. Using a transformation function equal to the cumulative distribution of $r$ produces an image whose gray levels have a uniform density, which implies an increase in the dynamic range of the pixels.

6. In order to be useful for digital image processing, equations should be formulated in discrete form:

$$
\mathrm{P}_{r}\left(r_{k}\right)=\frac{n_{k}}{n} \text { and } s_{k}=T\left(r_{k}\right)=\sum_{j=0}^{k} \frac{n_{j}}{n} \text {, where } \mathrm{k}=0,1 \ldots \mathrm{L}-1
$$

where $r_{k}$ is the input intensity, $\mathrm{s}$ is the processed intensity, $k$ is the intensity range (e.g. $0.0-1.0), n$ is the frequency of intensity $j, n$ is the sum of all frequencies 
7. A plot of $P_{r}\left(r_{k}\right)$ versus $r_{k}$ is actually a histogram, and the technique used for obtaining a uniform histogram is known as histogram equalization or histogram linearization [9] [18] [19].

\section{Noise Removal/Reduction}

We suggest the following methods, as defined by Zhou et al [1] and Gonzalez \& Woods [18], to preprocess a noisy image:

I. If the noise consists of fine speckle, try a median filter. It should be tried in a small neighborhood first, and then for larger neighborhoods. Always evaluate the effectiveness of the noise removal and the effect on features of interest.

To run a median filter:

a. Consider each pixel in the image

b. Sort the neighboring pixels into order based upon their intensities

c. Replace the original value of the pixel with the median value from the list.

II. If the speckle is mainly dark or light, try a morphological operation such as grey level closing or opening with a cylinder or sphere respectively [1]

III. If you have a lot of noise, experiment with wavelet thresholding. The Daubechies-6 and Daubechies-8 wavelets are fairly effective and straightforward to use.

IV. If the image has uneven illumination background, try low-pass filter.

V. If noise feature are not all small scale speckle, try alternating sequential morphological filters. Note that you have to carefully evaluate a range of filter size.

VI. If you intend to process an image with a threshold, try filtering before and after thresholding to determine which is most effective.

VII. If none of the above is effective, it may be useful to try morphological operations which filter objects on the basis of size or shape criteria [1]. These need to be used with care to avoid affecting features of interest.

VIII. If there is background articles/noise across the sample, try top-hat filter.

\section{Thresholding}

The optimum global thresholding technique is the Otsu method [9] [10] [11]. Otsu's method is used to automatically perform histogram shape-based image thresholding [9]. The algorithm assumes that the image to be thresholded contains two classes of pixels or bi-modal histogram (e.g. foreground and background) then calculates the optimum threshold separating those two classes so that their combined spread (intra-class variance) is minimal The method is optimum in the sense that it maximizes the between-class variance, a wellknown measure used in statistical discriminant analysis [9] ]10] [11].

The Otsu's algorithm [9] is given below:

i. Compute the normalized histogram of the input image. Denote the components of the histogram by $p_{i}, i=0,1,2 \ldots L-1$.

ii. Compute the cumulative sums, $P_{i}(k)$, for $k=0,1,2 \ldots L-1$, using

$P_{i}(k)=\sum_{i=0}^{k} p_{i}$

iii. Compute the cumulative means, $m(k)$, for $k$ $=0,1,2 \ldots L-1$, using

$m(k)=\sum_{i=0}^{k} i p_{i}$

iv. Compute the global intensity mean, $m_{G}$, using

$m_{G}=\sum_{i=0}^{L-1} i p_{i}$

v. Compute the between-class variance, $\sigma_{B}^{2}(k)$, for $k=0,1,2 \ldots L-1$, using

$$
\sigma_{B}^{2}(k)=\frac{\left[m_{G} P_{1}(k)-m(k)\right]^{2}}{P_{1}(k)\left[1-P_{1}(k)\right]}
$$

vi. Obtain the Otsu threshold, $k^{*}$, as the value of $k$ for which

$\sigma_{B}^{2}(k)$ is maximum. If the maximum is not unique, obtain

$k^{*}$ averaging the values of $k$ corresponding to the various maxima detected.

vii. Obtain the separability measure, $\eta^{*}$, by evaluating

$(k)=\frac{\sigma_{B}^{\mathbb{Z}}(k)}{\sigma_{G}^{\mathbb{Z}}}$ at $k=k^{*}$

where $\sigma_{G}^{2}$ is the global variance [i.e., the intensity variance of all pixels in the image]

$\sigma_{G}^{2}=\sum_{i=0}^{L-1}\left(i-m_{G}\right)^{2} P_{i}$

\section{Edge Detection}


For detection of edges in medical images, we have proposed the following algorithm:

Step1: First take an input image. Step2: Apply edge detection technique.

Step3: Apply direction if necessary (Horizontal/Vertical, both).

Step4: Display results.

Step5: End.

The X-Ray image is the input image. We have different edge detection techniques; we propose to compare five (5) of these techniques. These detectors are:

i. Sobel operators: The computation of the partial derivation in gradient may be approximated in digital images by using the Sobel operators [2] [20] which are shown in the masks in Figure 7 below:

\begin{tabular}{|l|l|l|}
\hline-1 & 0 & 1 \\
\hline-2 & 0 & 2 \\
\hline-1 & 0 & 1 \\
\hline
\end{tabular}

\begin{tabular}{|c|c|c|}
\hline 1 & 2 & 1 \\
\hline 0 & 0 & 0 \\
\hline-1 & -2 & 1 \\
\hline
\end{tabular}

Figure 7: The Sobel masks [20]

These two masks together with any of the equations:

$|\nabla f|=\sqrt{G_{x}^{2}+G_{y}^{2}}$

$|\nabla f|=\left|G_{x}\right|+\left|G_{y}\right|$

are used to obtain the gradient magnitude of the image from the original [2] [20].

ii. Roberts cross operator: The Roberts Cross operator [2] [20] performs a simple, quick to compute, 2-D spatial gradient measurement on an image. It thus highlights regions of high spatial frequency which often correspond to edges. In its most common usage, the input to the operator is a gray scale image, as is the output. Pixel values at each point in the output represent the estimated absolute magnitude of the spatial gradient of the input image at that point [2] [20]. Roberts Cross convolution mask is shown in Figure 8.
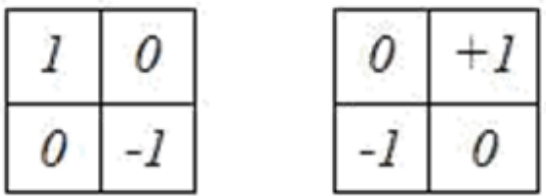

Figure 8: Roberts cross convolution mask [20]

iii. Prewitt operator: The Prewitt operator measures two components. The vertical edge component is calculated with kernel $G x$ and the horizontal edge component is calculated with kernel $G y \cdot|G x|+|G y|$ give an indication of the intensity of the gradient in the current pixel [2] [20]. The Prewitt operator is shown in Figure 9.

\begin{tabular}{|l|l|l|}
\hline-1 & 0 & 1 \\
\hline-1 & 0 & 1 \\
\hline-1 & 0 & 1 \\
\hline
\end{tabular}

Gx

\begin{tabular}{|c|c|c|}
\hline 1 & 1 & 1 \\
\hline 0 & 0 & 0 \\
\hline-1 & -1 & -1 \\
\hline
\end{tabular}

Gy
Figure 9: Prewitt Mask [20]

iv. Laplacian operator: The Laplacian of an image $f(x, y)$ is a second order derivative defined as:

$\nabla^{2} f=$

The digital implementation of the Laplacian function [2] [20] is usually made through the mask in Figure 10 below:

\begin{tabular}{|c|c|c|}
\hline 0 & -1 & 0 \\
\hline-1 & 4 & -1 \\
\hline 0 & -1 & 0 \\
\hline
\end{tabular}

Figure 10: The Laplacian masks [20]

The Laplacian is usually used to establish whether a pixel is on the dark or light side of an edge.

v. Canny edge detector: Canny technique [2] [20] is very important method to find edges by isolating noise from the image before find edges of image, without affecting the features of the edges in the image and then applying the tendency to find the edges and the critical value for threshold.

The algorithmic steps for canny edge detection technique are follows:

a. Convolve image $\mathrm{f}(r, c)$ with a Gaussian function to get smooth image $\mathrm{f}^{\wedge}(r, c)$.

$$
\mathrm{f}^{\wedge}(r, c)=\mathrm{f}(r, c)^{*} \mathrm{G}(r, c, 6)
$$

b. Apply first difference gradient operator to compute edge strength then edge magnitude and direction are obtain as before.

c. Apply non-maximal or critical suppression to the gradient magnitude.

d. Apply threshold to the non-maximal 
suppression image.

\section{Active contour model}

Active contours or snakes are computer generated curves that move within the image to find object boundaries under the influence of internal and external forces [2] [15].

This procedure is as follows:-

1. Snake is placed near the contour of Region of Interest (ROI). A snake in the image can be represented as a set of $n$ points.

$$
V_{i}=\left(x_{i}, y_{i}\right)
$$

where $i=0 \ldots n-1$

2. During an iterative process due to various internal and external forces within the image, the Snake is attracted towards the target. These forces control the shape and location of the snake within the image.

3. An energy function is constructed which consist of internal and external forces to measure the appropriateness of the Contour of ROI, Minimize the energy function (integral), which represents active contour's total energy, The internal forces are responsible for smoothness while the external forces guide the contours towards the contour of ROI. The energy function is given as:

$$
\begin{gathered}
E_{\text {snake }}^{*}=\int_{0}^{1} E_{\text {snake }}(v(s)) d s \\
=\int_{0}^{1}\left(E_{\text {internel }}(v(s))+E_{\text {image }}(v(s))+E_{\text {con }}(v(s))\right) d s
\end{gathered}
$$

$$
\begin{gathered}
\text { where } E_{\text {external }}=E_{\text {image }}+E_{\text {con }} \\
E_{\text {internal }}=E_{\text {cont }}+E_{\text {curv }}
\end{gathered}
$$

Eexternal represents the internal energy of the spline (snake) due to bending, Eimage denotes the image forces acting on spline and Econ serves as external constraint forces introduced by user. The combination of Eimage and Econ can be represented as Eexter, that denote the external energy acting on the spline. Econt denotes the energy of the snake contour and Ecurv denotes the energy of the spline curvature [2] [15].

\section{Graphical Illustration of Results}

The algorithms considered in this section were all implemented with a test hand x-ray image [21] shown in Figure 11, on Matrix Laboratory 2017b, results are presented.

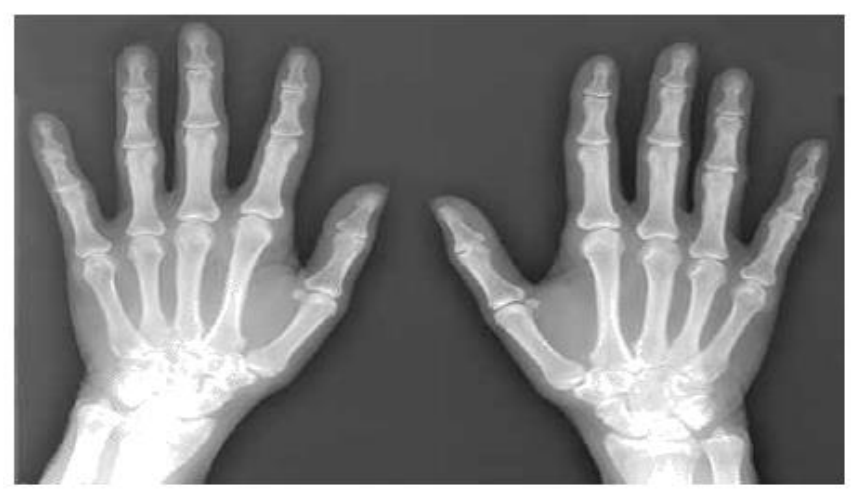

Figure 11: Test image (hand $x$-ray) [21]

1. Histogram generation: The histogram of the $\mathrm{x}-$ ray image is generated to determine if the intensity levels are widely spread [i.e. from 0 - 255]. The result obtained is shown in Figure 12 below.

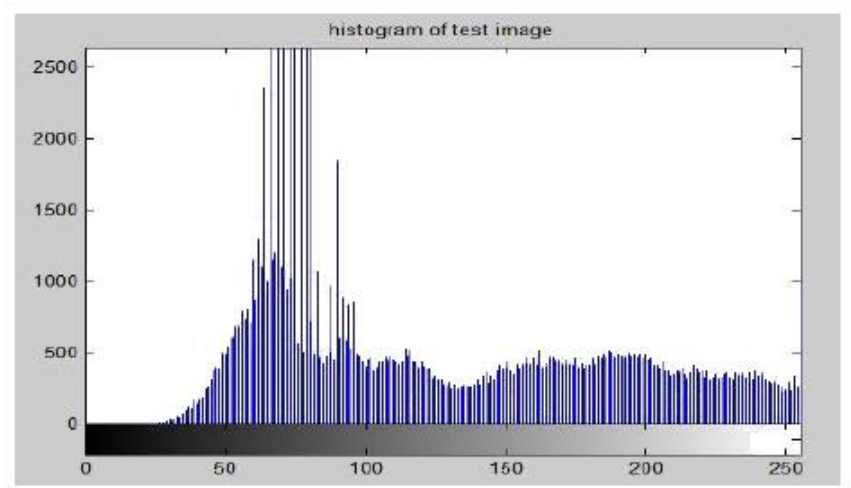

Figure 12: Histogram of the $x$-ray image

Image pre-processing: As shown in Figure 12 above, the intensity level is not widely dispersed i.e. not ranging from $0-$

255. To correct this image defect caused by uneven illumination in the image, the top-hat filter is applied and equalized. The result is shown in Figure 13 and Figure 14 below.

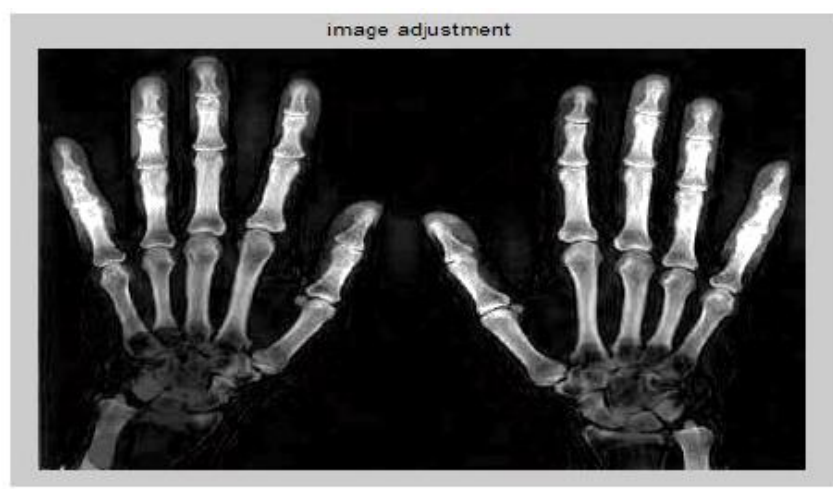

Figure 13: Pre-processed image 


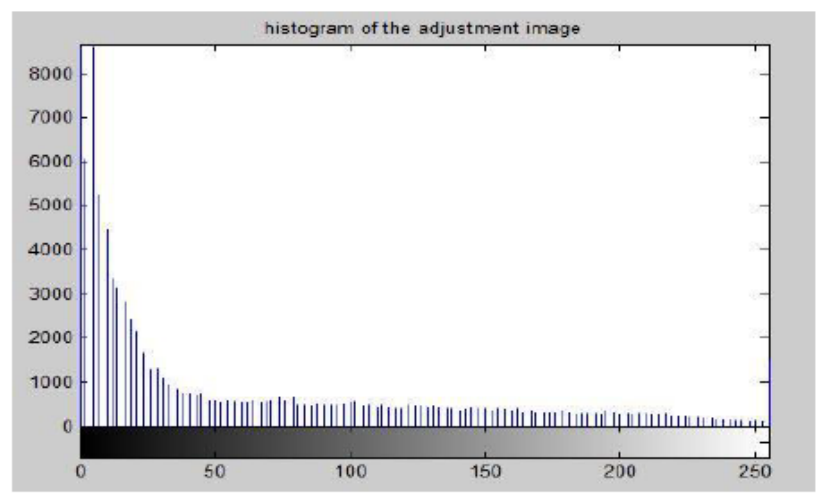

Figure 14: Histogram of the Pre-processed image

2. Thresholding: Upon successful correction of the image defect, thresholding technique is applied on the image and the result is shown in Figure 15 below.

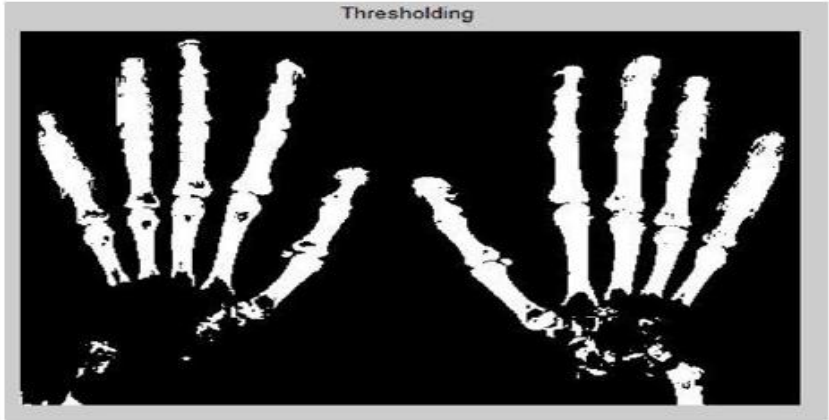

Figure 15: X-ray image segmentation using thresholding

3. Edge-based segmentation: Five different edge detectors were applied on the X-ray image and the results obtained are shown below. Figure 16 shows X-ray image segmentation using Prewitt operator. Figure 17 shows X-ray image segmentation using Sobel operator. Figure 18 shows X-ray image segmentation using Laplacian of Gaussian operator. Figure 19 shows X-ray image segmentation using Roberts operator. Figure 20 shows X-ray image segmentation using Canny operator.

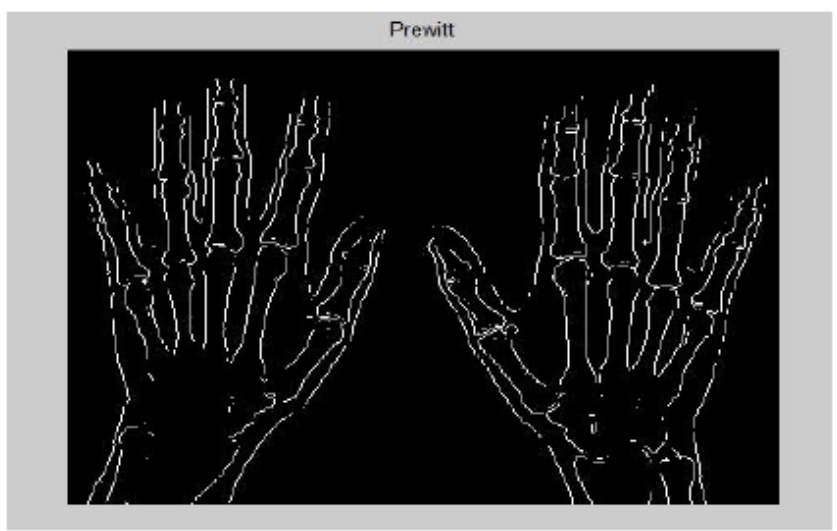

Prewitt operator

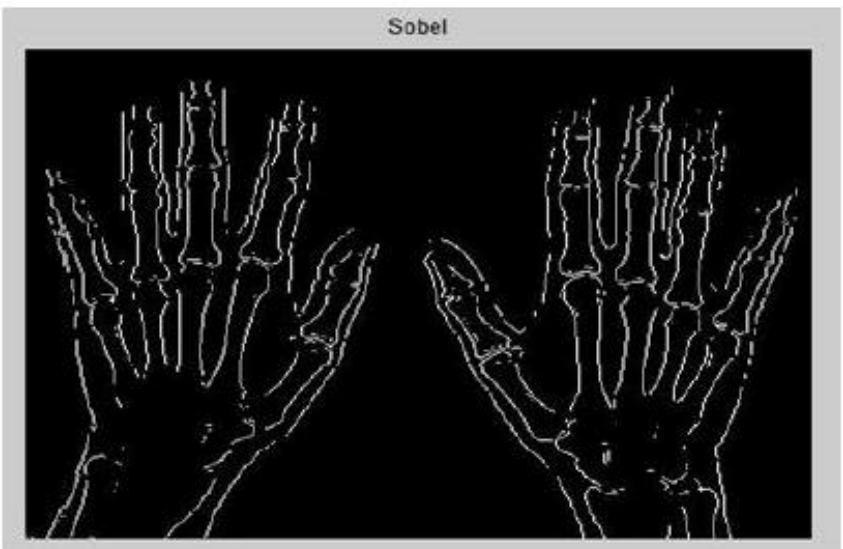

Figure 17: X-ray image segmentation using Sobel operator

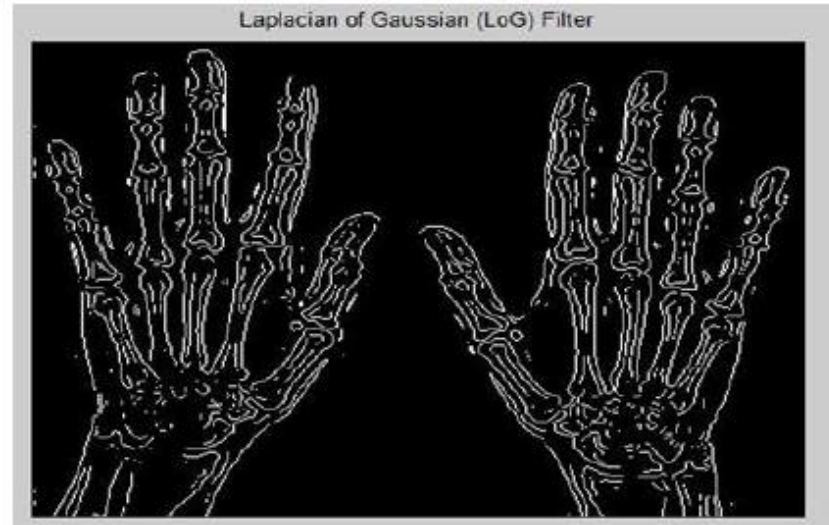

Figure 18: X-ray image segmentation using Laplacian of Gaussian operator

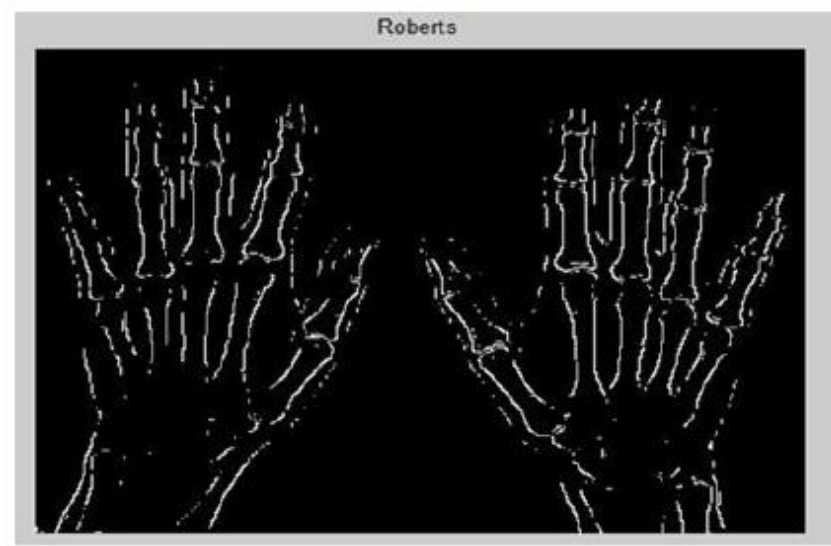

Figure 19: X-ray image segmentation using Roberts's operator

Figure 16: $X$-ray image segmentation using 


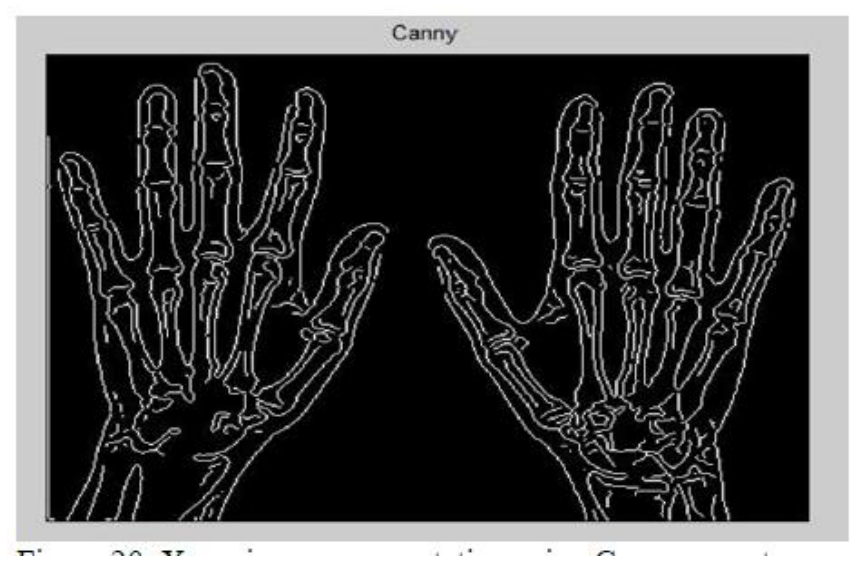

Figure 20: $X$-ray image segmentation using canny operator6. Conclusion and Future Work

From the visual inspection of the images obtained it can be seen that Prewitt filter gives a similar results as the Sobel and Roberts ; The results show that Canny is the most effective method, since it perfectly detects all the edges in the image (flesh and bones). But, if only the bone needs to be separated, a more general, robust and effective solution needs to be experimented and established.

In future, we want to continue to simulate the effects of more recent algorithms we have discovered. Abass and Mousa [22] developed an Edge detection method for medical images using Markov basis and Laplace filter. Bharodiya and Atul [23] developed an improved edge detection technique for X-ray images based on statistical range. We want to focus simulation on these newer techniques and compare with some existing methods. Also, we focus on building a Graphical user interface for the system design proposed to enable one-to-one interaction with the users of the system.

\section{References}

[1.] Huiyu Zhou, Jiahua $\mathrm{Wu}$ and Jianguo Zhang (2010). "Digital Image Processing-Part II", 2nd edition, Ventus Publishing.

[2.] Stolojescu-Crisan C. and Holban S.(2013) A Comparison of X-Ray Image Segmentation Techniques. Advances in Electrical and Computer Engineering, August.

[3.] Peng T.T. (2002), "Detection of Femur Fractures in X-ray images", $\mathrm{PhD}$ thesis, National University of Singapore.

[4.] Lim S.E., Y. Xing, Y. Chen, W. K. Leow, T. S. Howe, and M. A. Png (2004). "Detection of Femur and Radius Fractures in X-Ray Images." , 2nd International Conference on Advances in Medical Signal and Information.
[5.] V. L. F. Lum, W. K. Leow, Y. Chen, T. S. Howe, and M. A. Png, "Combining classifiers for bone fracture detection in $\mathrm{X}$ ray Images",

[6.] Sezgin M. and B. Sankur (2004). "Survey over image thresholding techniques and quantitative performance evaluation", Journal of Electronic Imaging.

[7.] Feng D. (2006), "Segmentation of bone structures in X-ray images", $\mathrm{PhD}$ thesis, School of computing National University of Singapore.

[8.] Long M. (2008). "Segmentation of Body Regions and Airways in Full-Body Digital Paediatric X-Ray Images", Master's thesis, University of Cape Town, August.

[9.] MathWorks. MATLAB Help, 2017b.

[10.] Bhargavi P.M., Mayee V.S.K., Manaswini T. and Manvitha S. (2015). "A comparison of Image Segmentation Techniques, OTSU and Watershed for X-Ray Images", International Journal of Research in Engineering and Technology. Vol.04 Issue 04, April. eISSN: 2319-1163

[11.] Gonzalez R.C. and R. E. Woods (2001), "Digital Image Processing". Prentice Hall.

[12.] Wu Z. and R. Leahy (1993), "An optimal graph theoretic approach to data clustering:Theory and its application to image segmentation," IEEE Transactions on Pattern Analysis and Machine Intelligence, vol. 15, no. 11, pp. 1101-1113, November.

[13.] Ren X. and J. Malik (2003), "Learning a classification model for segmentation," in International Conference on Computer Vision, Nice, France, October. Ajayi , IJECS Volume ... Issue ... June, 2020 Page No.01-02 Page 9

[14.] Brown M.S., M. F. Mcnitt-Gray, J. G. G. Nicholas J. Mankovich, J. Hiller, L. S. Wilson, and D. R. Aberle (1997), "Method for segmenting chest CT image data using an anatomical model: Preliminary results," IEEE Transactions on Medical Imaging, vol. 16, no. 6, pp. 828-839.

[15.] Kass M., A. Witkin, and D. Terzopoulos (1987), "Snakes: Active contour models," Int'1 J. Computer Vision, pp. 321-331.

[16.] Sethian J.A. (1999), "Level Set Methods and Fast Marching Methods". Evolving Interfaces in Computational Geometry, Fluid Mechanics, Computer Vision, and Materials Science. Cambridge University 
Press.

[17.] Cootes T,F., A. Hill, C. J. Taylor, and J.Haslam (1994), "The use of active shape models for locating structures in medical images," Image and Vision Computing, vol. 12 , no. 6, pp. 355-366, July.

[18.] Gonzalez R.C. and R. E. Woods (2002), "Digital Image Processing". 2nd edition,Addison-Wesley Publishing Company, Reading, MA, 2002.

[19.] Namee B.M. (2008). "Image Enhancement (Histogram Processing) ppt."

[20.] Salem Saleh Al-amri, Dr. N.V Kalyankar and Dr. S.D Khamitkar (2010),"Image Segmentation by using Edge detection", International journal on computer science and enginerring, volume 2, No. 03, 2010, pg. $804-807$

[21.] Bone X-Ray Available at http://radiologyinfo.org/en/amp/bonerad.htm 1. Last accessed March 2020.

[22.] Abbass H.H. and Mousa Z.R. (2017). "Edge detection of medical images using Markov basis", Applied Mathematical Sciences 11(37):1825-1833. July. DOI: 10.12988/ams.2017.75160

[23.] Bharodiya Anil and Atul M.G.(2019). "An improved edge detection algorithm for XRay images based on the statistical range", Heliyon Volume 5, Issue 10, October. Available at: https://www.sciencedirect.com/science/articl e/pii/S2405844019364035. Last accessed: April 2020.

\section{Author Profile}

Ajayi Adedoyin Olayinka received the B.Sc. in Computer Science from the Federal University of Agriculture, Abeokuta, Nigeria in 2007, and Masters and Ph.D. degrees in Computer Science from the Federal University of Technology, Akure, Nigeria, in 2012 and 2016, respectively. He majors in Wireles Sensor Network Security.

Tawose Olamide Timothy received the B.Sc. in Computer Science from Ekiti State University, Ado-Ekiti, Nigeria in 2014, and Master's degree from the University of Bradford, United Kingdom in 2017. He is a Ph.D. candidate in the University of Nevada, Reno, U.S.A. Adetolaju Olu Sunday received Masters in Information Technology from Obafemi Awolowo University, Ile-Ife, Nigeria. He is a Ph.D. candidate in Ekiti State University, Ado-Ekiti, Nigeria. 